\title{
New Algorithms for Blind Block Synchronization in Zero-Padding Systems
}

\author{
Borching Su \\ Department of Electrical Engineering \\ California Institute of Technology \\ Pasadena, California 91125 \\ Email: borching@systems.caltech.edu
}

\author{
P. P. Vaidyanathan \\ Department of Electrical Engineering \\ California Institute of Technology \\ Pasadena, California 91125 \\ Email: ppvnath@systems.caltech.edu
}

\begin{abstract}
Blind channel identification using linear redundant filterbank precoders (LRP) has been studied extensively in the literature. Most methods are proposed based on the assumption that block synchronization is perfect. In practice, a blind block synchronization algorithm must be used to justify this assumption. This paper studies the blind block synchronization problem in systems using a zero-padding (ZP) precoder. A previously reported method is reviewed and a new approach for the problem is proposed. Generalized versions of both approaches are then developed using a parameter called repetition index. Simulation results show that when the repetition index is chosen to be greater than unity, the block synchronization error rate performance of the proposed algorithm has a significant improvement over the previously reported method. ${ }^{1}$
\end{abstract}

Index Terms - Frame synchronization, Blind Block Synchronization, Zero-Padding, Repetition Index.

\section{INTRODUCTION}

Blind channel identification using linear redundant filter bank precoders (LRP) has been studied extensively in the literature [1]-[6]. Besides a constant bandwidth overhead introduced in each block, a blind channel estimation method usually requires very little extra bandwidth to perform channel estimation. Most existing blind estimation methods for LRPs assume the boundaries of blocks of the received stream are perfectly known to the receiver. In practical applications, however, this assumption is usually not true since no extra known samples are transmitted. In this paper we study the problem of blind recovery of block boundaries for the received signal. In particular, we consider the problem on a block transmission system using a zero-padding (ZP) precoder. Scaglione et al. proposed the first blind block synchronization algorithm in [1] as well as two blind channel identification/equalization algorithms. The blind equalization algorithm uses a matrix composed of elements in received blocks which is rank deficient in absence of noise. Now, the blind block synchronization algorithm exploits the fact that when the synchronization of received blocks is incorrect, the rank deficiency property of this matrix used for blind equalization is no longer valid.

More recently in the literature, Manton et al. pointed out that blind channel estimation can be done with fewer received blocks by repeated use of each block [2], [3]. This concept was later generalized by $\mathrm{Su}$ and Vaidyanathan [4], [5] using a parameter called repetition index. The repetition index idea can also be used for blind synchronization. This paper explores this idea. Another novelty is that the method is based on a subspace of dimension $L$ rather than one as in [1] (where $L$ is the channel order). This idea, combined with the repetition index, is shown to significantly improve the performance.

\footnotetext{
${ }^{1}$ Work supported in parts by the NSF grant CCF-0428326 and the Moore Fellowship of the California Institute of Technology.
}

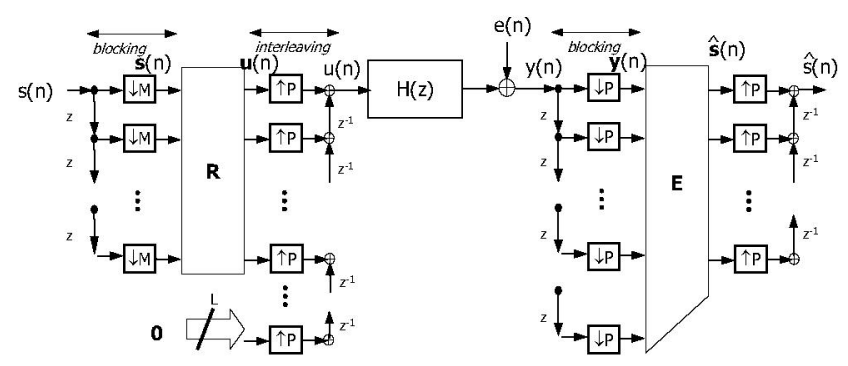

Fig. 1. A typical zero-padding system

The rest of the paper is organized as follows. Section II formulates the problem statement and briefly reviews a blind block synchronization algorithm proposed in [1]. In Section III we first propose a new approach for blind block synchronization and then develop the generalized versions of both algorithms using the concept of repetition index. Simulation results and discussions are presented in Section IV and conclusions are made in Section V.

\section{A. Notations}

Boldfaced lower case letters represent column vectors. Boldfaced upper case letters are reserved for matrices. Superscripts $T$ and ${ }^{\dagger}$ as in $\mathbf{A}^{T}$ and $\mathbf{A}^{\dagger}$ denote the transpose and transposeconjugate operations, respectively. $\mathbf{I}_{n}$ is the $n \times n$ identity matrix. All the vectors and matrices in this paper are in general complex-valued. If $\mathbf{v}=\left[\begin{array}{llll}v_{1} & v_{2} & \cdots & v_{m}\end{array}\right]^{T}$ is an $m \times 1$ vector, we use $\mathcal{T}_{n}(\mathbf{v})$ to denote the $(m+n-1) \times n$ full-banded Toeplitz matrix [7] whose first column is $\left[\begin{array}{cc}\mathbf{v}^{T} & \mathbf{0}_{(n-1) \times 1}^{T}\end{array}\right]^{T}$ and whose first row is $\left[\begin{array}{cc}v_{1} & 0_{1 \times(n-1)}\end{array}\right]$.

\section{Problem Formulation and Literature Review}

Figure 1 shows a typical zero-padding $(\mathrm{ZP})$ transceiver. The data samples, $s(n)$, are blocked into vectors $\mathbf{s}(n)$ of size $M$. The precoded vector, $\mathbf{u}_{z p}(n)$, of size $P=M+L$, is composed of an $M$-vector $\mathbf{u}(n)=\mathbf{R s}(n)$ followed by a zero segment of length $L$. The vector sequence $\mathbf{u}_{z p}(n)$ is then unblocked into a sample sequence $u(n)$ before sending to the channel. The channel is characterized as an $L$ th order FIR system $H(z)=\sum_{k=0}^{L} h_{k} z^{-k}$. We use an $(L+1)$-vector $\mathbf{h}$ to express the coefficients

$$
\mathbf{h}=\left[\begin{array}{llll}
h_{0} & h_{1} & \cdots & h_{L}
\end{array}\right]^{T} .
$$

At the receiver side, the received sample stream $y(n)$ is blocked into vectors $\mathbf{y}(n)$ of size $P$. Due to trailing zeros 


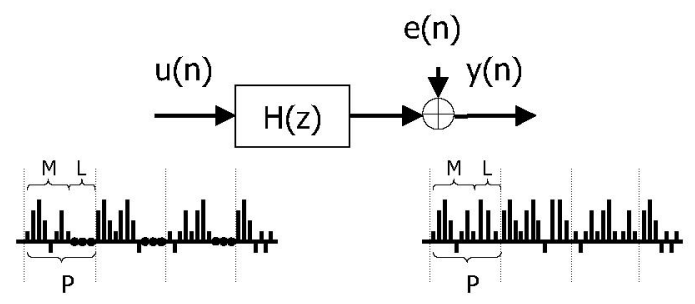

Fig. 2. Illustration of blind block synchronization problem in ZP systems

introduced in each block at the transmitter, the inter-block interference (IBI) is eliminated. The received blocks $\mathbf{y}(n)$, in absence of noise, can be written in terms of channel coefficients and transmitted blocks:

$$
\mathbf{y}(n)=\mathcal{T}_{M}(\mathbf{h}) \mathbf{u}(n)
$$

A blind channel estimation algorithm aims at estimating channel coefficients $\mathbf{h}$ using only observation of blocks $\mathbf{y}(n)$ without knowledge of $\mathbf{u}(n)$. In the literature, many algorithms [1]-[6] have been proposed based on the assumption that block boundaries of $y(n)$ are perfectly known to the receiver.

Suppose there is an unknown timing mismatch $d_{0} \in$ $[-P / 2, P / 2)$ between the transmitter and the receiver so that instead of $y(n)$, the signal $y\left(n-d_{0}\right)$ is received. The problem of interest is how we can recover $d_{0}$ without knowledge of transmitted data $\mathbf{u}(n)$ and the channel coefficients $\mathbf{h}$ so that all blind estimation methods [1]-[6] can proceed to work.

Without loss of generality and for convenience of presentation, we assume $d_{0}=0$ throughout the paper. Figure 2 illustrates the problem statement. We find that the signal $u(n)$ has clear block boundaries due to the presence of zero samples of length $L$ at the end of each block $\mathbf{u}_{z p}(n)$. However, due to the $L$ th order FIR channel $H(z)$, the received signal $y(n)$ does not have zero-segments and hence boundary lines can not be detected by looking for zero-samples in $y(n)$.

In Eq. (1), samples $\mathbf{y}(n)$ are collected as

$$
\mathbf{y}(n)=\left[\begin{array}{llll}
y(n P) & y(n P+1) & \cdots & y(n P+P-1)
\end{array}\right]^{T}
$$

when block synchronization is perfect. Now suppose the blocking is performed as if there were a timing mismatch $d \in[-P / 2, P / 2)$. Then the samples collected in the $n$th block will be

$\mathbf{y}^{(d)}(n)=\left[\begin{array}{llll}y(n P+d) & y(n P+d+1) & \cdots & y(n P+d+P-1)\end{array}\right]^{T}$

We review a blind synchronization method proposed in [1]. Suppose $N$ consecutive blocks are collected at the receiver with a timing mismatch of $d$ samples. Given any integer $d \in$ $[-P / 2, P / 2)$, define the $P \times N$ matrix as

$$
\mathbf{Y}_{N}^{(d)}=\left[\begin{array}{llll}
\mathbf{y}^{(d)}(0) & \mathbf{y}^{(d)}(1) & \cdots & \mathbf{y}^{(d)}(N-1)
\end{array}\right] .
$$

Let $\mathbf{J}_{n}$ denote an $n \times n$ square shift matrix

$$
\mathbf{J}_{n}=\left[\begin{array}{cc}
\mathbf{0}^{T} & 0 \\
\mathbf{I}_{n-1} & 0
\end{array}\right]
$$

and consider the $P \times N L$ matrix

$$
\mathcal{Y}_{N}^{(d)}:=\left[\begin{array}{llll}
\mathbf{Y}_{N}^{(d)} & \mathbf{J}_{P} \mathbf{Y}_{N}^{(d)} & \cdots & \mathbf{J}_{P}^{L-1} \mathbf{Y}_{N}^{(d)}
\end{array}\right]
$$

The following theorem has been proved regarding the rank of $\mathcal{Y}_{N}^{(d)} \mathcal{Y}_{N}^{(d) \dagger}$.

Theorem 1: Consider the noise-free situation and assume that $\mathbf{u}(n)$ is $\operatorname{rich}^{2}$. Then $\mathcal{Y}_{N}^{(d)} \mathcal{Y}_{N}^{(d) \dagger}$ has full rank $P$ when $d \neq 0$ and has rank $P-1$ when $d=0$.

Proof: See Theorem 4 in [1].

The block synchronization problem can thus be solved by finding the $d$ which makes the matrix $\mathcal{Y}_{N}^{(d)} \mathcal{Y}_{N}^{(d) \dagger}$ rank deficient. In practice when the noise is present, we use the a cost function defined as

$$
\lambda_{1}(d):=\min \left\{\text { eigenvalues of } \mathcal{Y}_{N}^{(d)} \mathcal{Y}_{N}^{(d) \dagger}\right\}
$$

The optimal $d$ can be chosen as $\hat{d}=\arg \min _{-\frac{P}{2} \leq d<\frac{P}{2}} \lambda_{1}(d)$.

The matrix $\mathcal{Y}_{N}^{(d)}$ defined in Eq. (3) was first proposed for blind direct channel equalization [1]. It was also exploited in the blind synchronization method of [1]. The blind synchronization algorithms we propose next will exploit properties from existing blind channel estimation algorithms in the literature [1], [4].

\section{PRoposed Methods}

\section{A. A New Approach for Blind Block Synchronization}

Consider the matrix $\mathbf{Y}_{N}^{(d)}$ defined in Eq. (2). When $d=0$, it had been shown that in absence of noise, $\mathbf{Y}_{N}^{(d)}$ has exactly $L$ left annihilators which are useful for blind channel estimation. In this section, we will exploit this property to develop a new approach for blind block synchronization.

Theorem 2: Consider the noise-free situation and assume that $\mathbf{u}(n)$ is rich. Then when $d=0, \mathbf{Y}_{N}^{(d)} \mathbf{Y}_{N}^{(d) \dagger}$ has exactly $L$ zero eigenvalues. When $d \neq 0, \mathbf{Y}_{N}^{(d)} \mathbf{Y}_{N}^{(d) \dagger}$ has strictly less than $L$ zero eigenvalues.

Proof: The proof is part of [8]. Please see [9] for ready access.

Using Theorem 2, a noise-free version of a blind block synchronization algorithm can be readily developed by finding the only $d \in[-P / 2, P / 2)$ which makes the $L$ smallest eigenvalues of $\mathbf{Y}_{N}^{(d)} \mathbf{Y}_{N}^{(d) \dagger}$ all zeros. When the noise is present, we use the sum of these $L$ eigenvalues as a cost function:

$$
\lambda_{2}(d):=\sum_{k=1}^{L}\left\{\text { the } k \text { th smallest eigenvalue of } \mathbf{Y}_{N}^{(d)} \mathbf{Y}_{N}^{(d) \dagger}\right\}
$$

Similarly, the estimated timing offset is chosen as the $d$ that minimizes $\lambda_{2}(d)$.

The new approach is conceptually simpler than the method reviewed in Section II in the way that it involves a much smaller matrix $\mathbf{Y}_{N}^{(d)}$, although there are $L$ eigenvalues, rather than one, needed to be computed. As we will see from the simulation results presented in Section IV, this new approach is actually less robust to noise than the method reviewed in Section II. However, we will soon demonstrate the value of presenting this approach when we develop the generalized algorithm of it next.

${ }^{2}$ We say a sequence of $M$-vectors $\mathbf{u}(n), n \geq 0$, is rich if there exists an
integer $N>M$ such that $[\mathbf{u}(0) \quad \mathbf{u}(1) \quad \ldots \quad \mathbf{u}(N-1)]$ has full row rank $M[1]$. 


\section{B. Generalized Versions of Blind Synchronization Algorithms}

We first introduce the concept of repetition index for blind channel estimation and then develop generalized versions of blind synchronization algorithms. It can be readily verified that the noiseless channel equation (1) implies

$$
\mathcal{T}_{Q}(\mathbf{y}(n))=\mathcal{T}_{M+Q-1}(\mathbf{h}) \mathcal{T}_{Q}(\mathbf{u}(n))
$$

where $Q$ is an arbitrary positive integer and the notation for the full-banded Toeplitz matrix $\mathcal{T}_{n}(\cdot)$ was defined in Section I-A. Note that Eq. (1) is a special case of Eq. (5) when $Q=$ 1. When $Q>1$, Eq. (5) is similar to Eq. (1) in the sense that $\mathcal{T}_{M+Q-1}(\mathbf{h})$ is still a full-banded Toeplitz matrix, except that the size is larger by $Q-1$. Note that $\mathcal{T}_{Q}(\mathbf{y}(n))$ is a $(P+Q-1) \times Q$ matrix. Focusing on a particular column of $\mathcal{T}_{Q}(\mathbf{y}(n))$ and the corresponding column of $\mathcal{T}_{Q}(\mathbf{u}(n))$ in Eq. (5), the resulting equation is exactly equivalent to the channel equation for a ZP system with a larger block size. Since there are $Q$ linearly independent columns in $\mathcal{T}_{Q}(\mathbf{y}(n))$, for every single received block $\mathbf{y}(n)$, we have equivalently $Q$ blocks for the "virtual" $\mathrm{ZP}$ system whose block size is $P+Q-$ 1. The parameter $Q$ is called the repetition index since each received block is repeatedly used $Q$ times. The concept of repetition index first arose in [5] for generalization of blind channel estimation algorithms. Now we will use it to develop generalized blind block synchronization algorithms.

As a generalization of Eq. (2), we define the $(P+Q-1) \times$ $Q N$ matrix

$\mathbf{Y}_{N, Q}^{(d)}=\left[\begin{array}{llll}\mathcal{T}_{Q}\left(\mathbf{y}^{(d)}(0)\right) & \mathcal{T}_{Q}\left(\mathbf{y}^{(d)}(1)\right) & \cdots & \mathcal{T}_{Q}\left(\mathbf{y}^{(d)}(N-1)\right)\end{array}\right]$

Also, generalizing (3), define the $(P+Q-1) \times Q N L$ matrix

$\mathcal{Y}_{N, Q}^{(d)}:=\left[\begin{array}{llll}\mathbf{Y}_{N, Q}^{(d)} & \mathbf{J}_{P+Q-1} \mathbf{Y}_{N, Q}^{(d)} & \cdots & \mathbf{J}_{P+Q-1}^{L-1} \mathbf{Y}_{N, Q}^{(d)}\end{array}\right]$.

A generalized version of the method reviewed in Section II is obtained by simply replacing $\mathcal{Y}_{N}^{(d)}$ in Eq. (4) with $\mathcal{Y}_{N, Q}^{(d)}$. It can be shown [8] that, as a generalization of Theorem 1, $\mathcal{Y}_{N, Q}^{(d)} \mathcal{Y}_{N, Q}^{(d) \dagger}$ has full rank $P+Q-1$ when $d \neq 0$ and has rank $P+Q-2$ when $d=0$. Note that by choosing $Q=1$ the generalized algorithm reduces to the original algorithm. We designate the generalized version as Algorithm 1 and summarize it below.

Algorithm 1:

1) Choose the repetition index $Q \geq 1$ and the number of collected blocks $N \geq P$.

2) Collect $(N+1) P$ consecutive received samples and form the matrix $\mathcal{Y}_{N, Q}^{(d)}$ as in Eq. (7) for each $d \in[-P / 2, P / 2)$.

3) Evaluate the cost function

$$
\lambda_{1, Q}(d):=\min \left\{\text { eigenvalues of } \mathcal{Y}_{N, Q}^{(d)} \mathcal{Y}_{N, Q}^{(d) \dagger}\right\}
$$

for each $d$ and decide the estimated timing offset $\hat{d}=$ $\arg \min _{-\frac{P}{2} \leq d<\frac{P}{2}} \lambda_{1, Q}(d)$.

Similarly, the algorithm proposed in Section III-A can be generalized by replacing $\mathbf{Y}_{N}^{(d)}$ with $\mathbf{Y}_{N, Q}^{(d)}$ defined in Eq. (6). We designate it as Algorithm 2 and summarize it as follows.

Algorithm 2:

1) Choose the repetition index $Q \geq 1$ and the number of collected blocks $N \geq P$.

2) Collect $(N+1) P$ consecutive received samples and form the matrix $\mathbf{Y}_{N, Q}^{(d)}$ as in Eq. (6) for each $d \in[-P / 2, P / 2$ ).
3) Perform eigen-decomposition on the matrix $\mathbf{Y}_{N, Q}^{(d)} \mathbf{Y}_{N, Q}^{(d) \dagger}$ and take the $L$ smallest eigenvalues $\sigma_{L,(d)}^{2} \geq \sigma_{L-1,(d)}^{2} \geq$ $\cdots \geq \sigma_{2,(d)}^{2} \geq \sigma_{1,(d)}^{2} \geq 0$.

4) Calculate the cost function $\lambda_{2, Q}(d):=\sum_{k=1}^{L} \sigma_{k,(d)}^{2}$, and decide the estimated timing offset $\hat{d}=$ $\arg \min _{-\frac{P}{2} \leq d<\frac{P}{2}} \lambda_{2, Q}(d)$.

\section{Simulation Results and Discussion}

In this section, we conduct simulations to compare the performance of Algorithms 1 and 2 under different repetition indices $Q=1,2,3$. In all simulations, the number of data samples per block is chosen as $M=16$ and the length of padding zeros per block is $L=4$ (which implies $P=20$ ). The number of blocks collected for the algorithms is $N=$ 20. The constellation of data samples is QPSK. Simulations are conducted with two different 4th order FIR channels. Channel 1 has zero locations at $(1.2,-0.9,0.7 j,-0.7)$ and Channel 2 has zero locations at $(0.8,-0.8,0.5 j,-0.5 j)$, which is a minimum-phase system. Note that the special case of Algorithm 1 with $Q=1$ is equivalent to the existing method proposed in [1] (SGB method).

\section{A. Noise-free Case}

We first apply the algorithms in absence of noise. Figures 3 and 4 show the plots for average values of $\lambda_{k, Q}(d), k=1,2$ for Channels 1 and 2, respectively. For a clearer view of the values of $\lambda_{k, Q}(d)$ in the neighborhood of $d=0$, a close-up window is put at the top of each plot. As expected, $\lambda_{k, Q}(d)=$ 0 when $d=0$ and are nonzero otherwise for all $k$ and $Q$. The robustness of Algorithm $k$ against noise perturbation for a specific $Q$ may be roughly evaluated by looking at the values $\Delta_{\text {left }}^{k, Q}:=\lambda_{k, Q}(-1)-\lambda_{k, Q}(0)$ and $\Delta_{\text {right }}^{k, Q}:=\lambda_{k, Q}(1)-\lambda_{k, Q}(0)$. For both channels, Algorithm 2 with $Q=3$ has the largest values of $\Delta_{\text {left }}^{k, Q}$ and $\Delta_{\text {right }}^{k, Q}$ among all cases, even though the very same algorithm with $Q=1$ has the smallest among all cases. This shows the potential benefit to system performance by using a large $Q$. Another noteworthy observation could be made for Channel 2. Both algorithms with $Q=1$ have a very small value of $\Delta_{\text {right }}^{k, Q}$. This situation could contribute to a high synchronization error rate in a noisy environment. However, $\Delta_{\text {right }}^{k, Q}$ increases dramatically when $Q>1$ for both algorithms. On the contrary, values of $\Delta_{\text {left }}^{k, Q}$ of Algorithm 2 for Channel 2 do not increase very much even when $Q=3$.

\section{B. Performance in Presence of Noise}

Now we test the performance of the proposed algorithms in the presence of noise. ${ }^{3}$ The additive noise $e(n)$ is white and Gaussian. Over 2000 independent realizations were performed to produce the simulation plots. Figures 5 and 6 show the blind block synchronization error rate versus the SNR at the channel output for Channels 1 and 2, respectively. We observe that when Algorithm 1 is used, increasing the repetition index $Q$ does not significantly improve the error rate performance except in a high SNR region $(>25 \mathrm{~dB})$ for Channel 2. For Algorithm 2, on the contrary, the performance of the case $Q=1$ is worse than the SGB method, but the performances

\footnotetext{
${ }^{3}$ In a private communication with the first author of [1], we confirmed that some plots (Figure $3(\mathrm{a})$-(c)) presented in the original work of [1] contain some minor errors in the scales of SNR levels. After correction, curves in Figure 3 (c) in [1] match perfectly with the blue curves shown in Figures 5 and 6.
} 


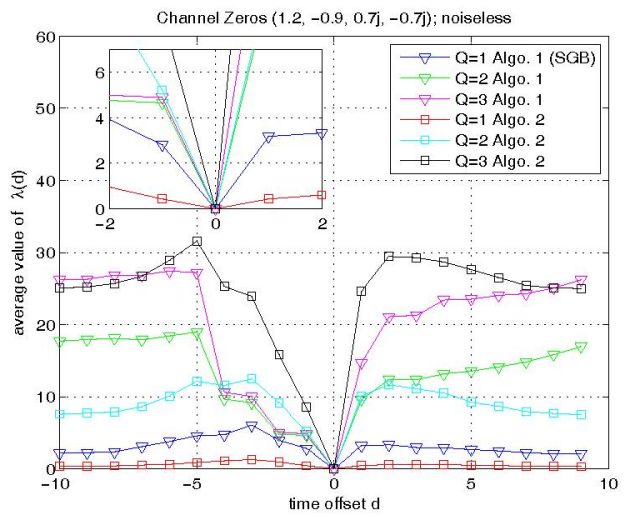

Fig. 3. Function $\lambda(d)$ v.s. time offset $d$ for a channel with zeros at $(1.2,-0.9,0.7 \mathrm{j},-0.7 \mathrm{j})$ in absence of noise.

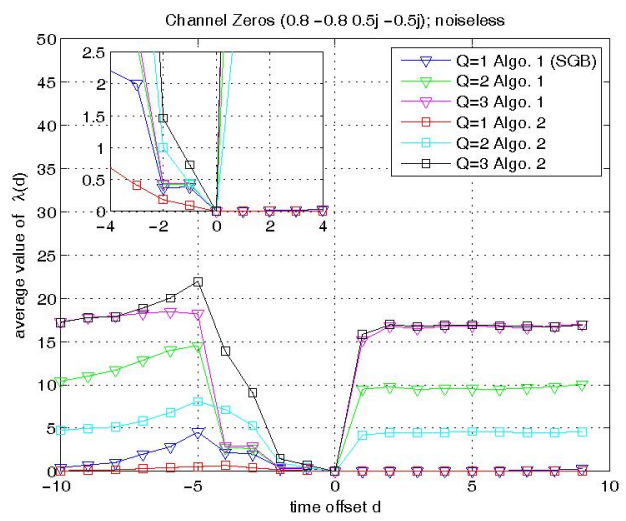

Fig. 4. Function $\lambda(d)$ v.s. time offset $d$ for a minimum-phase channel with zeros at $(0.8,-0.8,0.5 \mathbf{j},-0.5 \mathrm{j})$ in absence of noise.

for $Q=2,3$ have a significant improvement and outperform the SGB method by a large margin for both channels.

As a final comment, the performance curves for each algorithm are highly dependent on the channel zero locations. To achieve the same performance, the SNR level for Channel 2 must be much higher than that for Channel 1 (a difference of around $10 \mathrm{~dB}$ !). A more thorough study of performances of other different channels will be undertaken in the future.

\section{Conclusions}

In this paper we proposed two generalized algorithms for blind block synchronization in zero-padding (ZP) systems with a parameter called repetition index $(Q)$ which can be chosen as an arbitrary positive integer. In particular, a special case of Algorithm 1 with $Q=1$ reduces to a previously reported method proposed in [1]. Simulation results over two different LTI channels show that Algorithm 2, with a choice of $Q>$ 1 , has a significantly better performance than the previously reported method.

In the future, performance evaluation of the proposed algorithms for time-varying channels will be important for a more realistic scenario. A theoretical analysis of the system performance is also of interest. Furthermore, it would be of great importance to develop counterparts of these algorithms in cyclic prefix (CP) systems since most currently popular standards (e.g., OFDM, SC-CP, etc.) use cyclic prefix (CP) rather than zero-padding $(\mathrm{ZP})$ precoders.

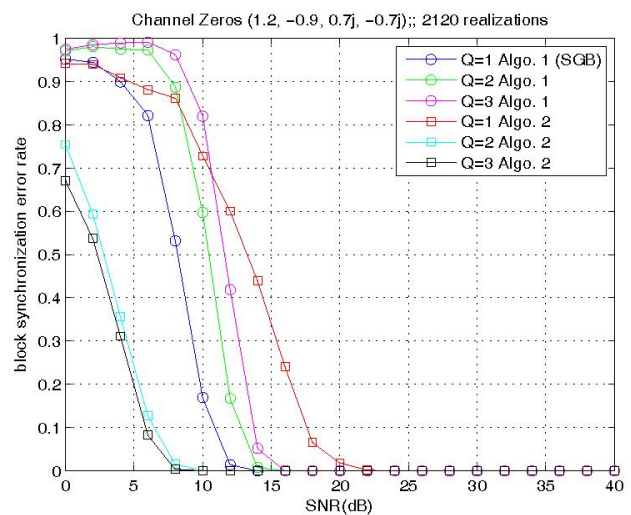

Fig. 5. Blind block synchronization error rate performance for a channel with zeros at $(1.2,-0.9,0.7 \mathrm{j},-0.7 \mathrm{j})$.

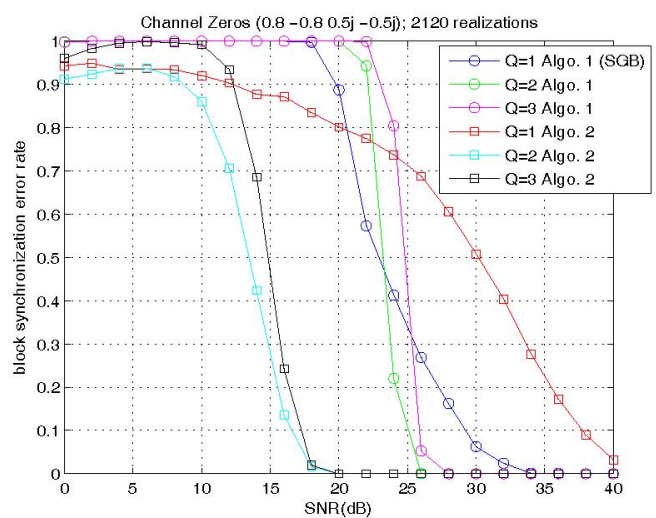

Fig. 6. Blind block synchronization error rate performance for a channel with zeros at $(0.8,-0.8,0.5 \mathrm{j},-0.5 \mathrm{j})$.

\section{ACKNOWLEDGMENT}

The authors would like to thank Prof. Anna Scaglione for sharing her program and for helpful discussions on the blind synchronization algorithm proposed in [1].

\section{REFERENCES}

[1] A. Scaglione, G. B. Giannakis, and S. Barbarossa, "Redundant filter bank precoders and equalizers Part II: Unification and Optimal Designs", IEEE Trans. SP, vol. 47, pp. 2007-2022, Jul. 1999.

[2] J. H. Manton and W. D. Neumann, "Totally Blind Channel Identification by Exploiting Guard Intervals", Systems and Control Letters, vol. 48, no. 2, pp. 113-119, 2003.

[3] D. H. Pham and J. H. Manton, "A Subspace Algorithm for Guard Interval Based Channel Identification and Source Recovery Requiring Just Two Received Blocks", Proc. of ICASSP 2003, pp. 317 - 320, Hong Kong, China

[4] B. Su and P. P. Vaidyanathan, "A Generalized Algorithm for Blind Channel Identification with Linearly Redundant Precoding," in print, EURASIP Journal on Applied Signal Processing Special Issue on Multirate Systems.

[5] B. Su and P. P. Vaidyanathan, "A Generalization of Deterministic Algorithm for Blind Channel Identification with Filter Bank Precoders", in Proc. ISCAS 2006, Kos Island, Greece, May 2006.

[6] S. Zhou and G. B. Giannakis, "Finite-alphabet based channel estimation for OFDM and related multicarrier systems," IEEE Trans. Commun., vol. 49, pp. 1402-1414, Aug. 2001.

[7] R. A. Horn and C. R. Johnson, Matrix Analysis, Cambridge University Press, 1996.

[8] B. Su and P. P. Vaidyanathan, "Blind Block Synchronization Algorithms using Redundant Filterbank Precoders," In preparation.

[9] Proof of Theorem 2, http://www.systems.caltech.edu/borching/proof.pdf (for reviewers only). 